\title{
Modeling of Satellite-Earth Link Channel and Simulating in Space-Ground Integrated Network
}

\author{
Beishan Wang ${ }^{1, *}$, Qi Guo ${ }^{2}$ \\ ${ }^{1,2}$ School of Information and Electronics, Beijing Institute of Technology, Beijing, China \\ Abstract
}

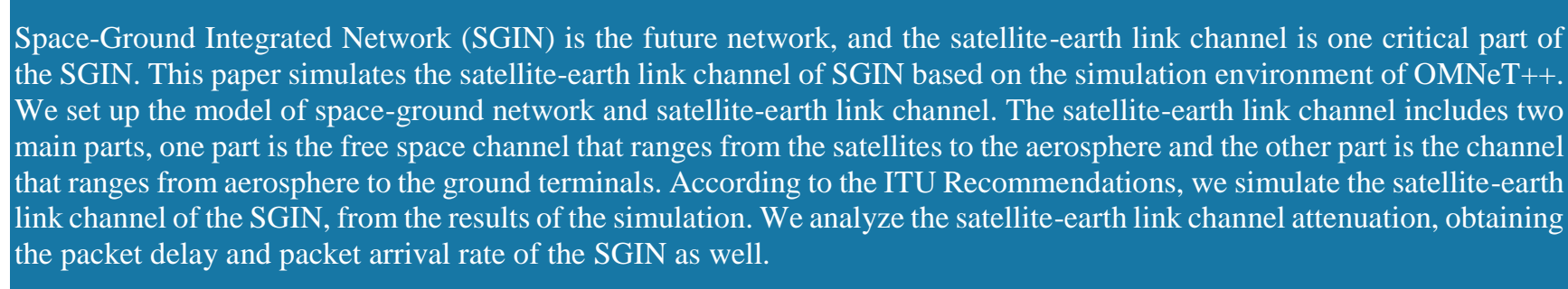

Keywords: satellite-earth Link channel, Space-Ground Integrated Network, OMNeT++ network simulation, packet delay, packet arrival rate.

Received on 15 August 2017; accepted on 2 November 2017; published on 8 December 2017

Copyright (C) 2017 Beishan Wang and Qi Guo, licensed to EAI. This is an open access article distributed under the terms of the Creative Commons Attribution license (http://creativecommons.org/licenses/by/3.0/), which permits unlimited use, distribution and reproduction in any medium so long as the original work is properly cited.

doi:10.4108/eai.8-12-2017.153458

\section{Introduction}

With the development of the STEM, especially the wireless communications. More and more information and data are propagated by satellite-communication, because there are amount of amazing advantages by using satellite to relay the signal, such as the wide coverage area, the long distance of communication and the wide frequency band. While the decreasing SNR and long-time delay since the longer distance between terminal and satellite are something we do not expect, so the research of satellite-earth link channel is worth for all satellite communication.

Muti-network fusion is one of the trends of future network, in the near future, we may build a unified network which contain the space satellite network and the ground internet. It will connect the earth with the outer space, and also is the foundation of IoT(Internet of Things). Therefore, the research of space-ground integrated network is important and worthful. In the propagation link, the satellite-earth link channel is one critical part of the SGIN $[1,2]$.

\section{Simulation of Satellite-Earth Link Channel}

\section{1. $\mathrm{OMNeT++}$}

There are several simulator of open source integrated development environment, such as NS2, OPNET. We choose
OMNET++ software to simulate the satellite-earth channel model. OMNeT++ is the discrete event simulator which is more friendly-using and powerful to model wire communication network, wireless communication network and protocol simulation. In general, it can be used in any system simulation and modeling that can be solved by discrete event method [3, 4].

\subsection{Build the Structure of the Space-Ground Network}

Based on the orbit parameters of the satellites and the coordinates of the ground stations, we write the NED files and $\mathrm{C}++$ and $\mathrm{h}$ files to set up the simulation space-ground network in the OMNeT++, the $2 \mathrm{D}$ view of the space-ground network is the following screenshot Figure 1.

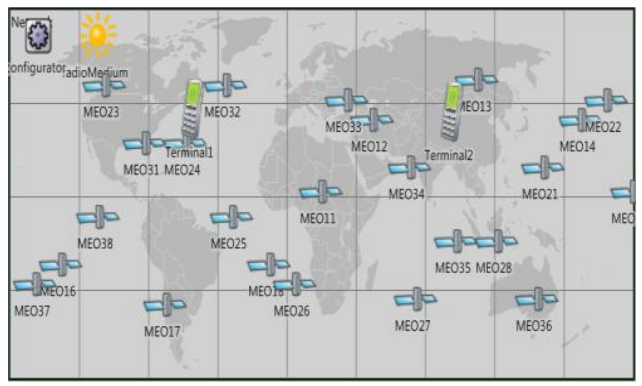

Figure 1. 2D view of the Space-Ground network.

*Corresponding author. Email: beishanwang@bit.edu.cn 


\subsection{Build the Instance of the Satellite-earth Link Channel}

The satellite-earth link channel includes two main parts, one part is the free space channel that ranges from the satellites to the aerosphere and the other part is the channel that ranges from aerosphere to the ground terminals which mainly affected by three critical factors, the distance, the rainfall and the gas molecules characteristic frequency attenuation. The satellite-earth link channel attenuation is given by the following Figure 2 .

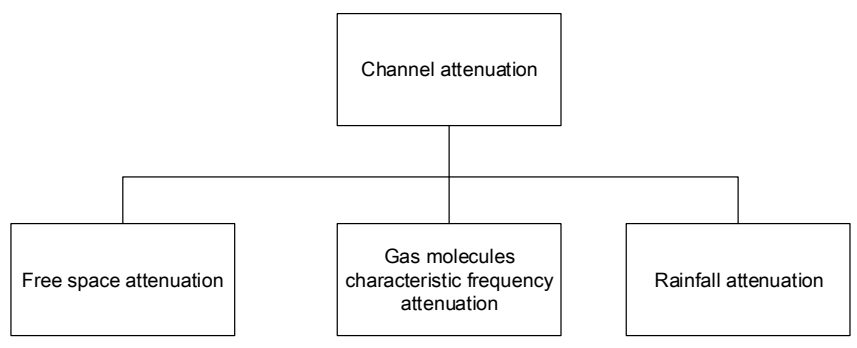

Figure 2. The satellite-earth link channel attenuation.

According to the ITU standard of channel Recommendation ITU-R P.618-10, and Recommendation ITU-R P.676-9, we build the instance of the satellite-earth link channel. The view of the instance of the satellite-earth link channel is given by the following Figure 3.

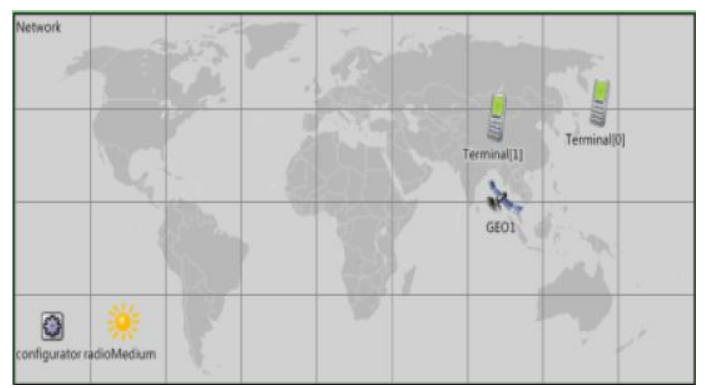

Figure 3. The view of the instance of the satellite-earth link channel.

The free space attenuation

The free space attenuation is easy to be derived from the equation of antenna. The attenuation $F$ is given by the following equations:

$$
F=(\lambda / D) / 16 \pi^{2} L
$$

Where:

$$
\begin{aligned}
& \lambda: \text { wavelength } \\
& d: \text { distance } \\
& L: \text { system loss. }
\end{aligned}
$$

The gas molecules characteristic frequency attenuation

The gas molecules characteristic frequency attenuation which is mainly determined by steam and dry air can be estimated by algorithms. The gas molecules characteristic frequency attenuation $\gamma_{o}(\mathrm{~dB} / \mathrm{km})$ is given by the following equations with the different frequency interval [5].

For $f \leq 54 \mathrm{GHz}$ :

$$
\left.\gamma_{o}=\frac{7.2 r_{t}^{2.8}}{f^{2}+0.34 r_{p}^{2} r_{t}^{1.6}}+\frac{0.62 \xi_{3}}{(54-f)^{1.16 \xi_{1}}+0.83 \xi_{2}}\right\rfloor f^{2} r_{p}^{2} \times 10^{-3} .
$$

For $54 \mathrm{GHz}<f \leq 60 \mathrm{GHz}$ :

$$
\gamma_{o}=\exp \left|\begin{array}{l}
\frac{\ln \gamma_{54}}{24}(f-58)(f-60) \\
\left\lfloor-\frac{\ln \gamma_{58}}{8}(f-54)(f-60)\right. \\
\left\lfloor+\frac{\ln \gamma_{60}}{12}(f-54)(f-58)\right.
\end{array}\right| .
$$

For $60 \mathrm{GHz}<f \leq 62 \mathrm{GHz}$ :

$$
\begin{aligned}
& \gamma_{o}=\gamma_{60}+\left(\gamma_{62}-\gamma_{60}\right) \frac{f-60}{2} . \\
& \phi\left(r_{p}, r_{t}, a, b, c, d\right)=r_{p}^{a} r_{t}^{b} e^{c\left(1-r_{p}\right)+d\left(1-r_{t}\right)} .
\end{aligned}
$$

Where:

$$
\begin{aligned}
& f: \text { frequency } \\
& r_{p}: r_{p}=p_{t o t} / 1013, \text { where } p_{\text {tot }} \text { represents total air } \\
& \quad \text { pressure } \\
& r_{t}: r_{t}=288 /(273+t) \\
& p: \text { pressure } \\
& t: \text { temperature. }
\end{aligned}
$$

\section{The rainfall attenuation}

The rainfall attenuation which is determined by rainfall can be estimated by the following geometry method, and there are so many correlation parameters in the calculation which we can obtain from the real physical world [6].

The diagram is presented in Figure 4. 


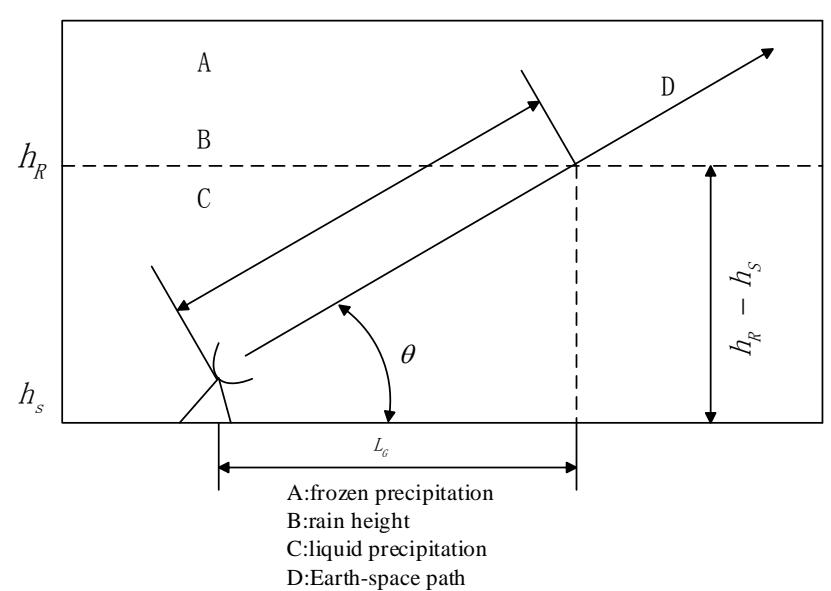

Figure 4. Diagram of an Earth-space path.

Step 1: Determine the rain height $h_{R}$, which can be found in P.839.

Step 2: Compute the parameter $L_{s}$

For $\theta \geq 5^{\circ}$

$$
L_{s}=\frac{\left(h_{R}-h_{s}\right)}{\sin \theta} .
$$

For $\theta<5^{\circ}$

$$
L_{s}=\frac{2\left(h_{R}-s_{s}\right)}{\left(\sin ^{2} \theta+\frac{2\left(h_{R}-h_{s}\right)}{R_{e}}\right)^{1 / 2}+\sin \theta} \cdot
$$

If $h_{R}-h_{S} \leq 0$ the attenuation is equal to 0 and the estimation is finished.

Step 3: Compute the parameter $L_{G}$

$$
L_{G}=L_{S} \cos \theta \text {. }
$$

Step 4: If $R_{0.01}=0$ the attenuation is equal to 0 and the estimation is finished.

Step 5: Compute the parameter $\gamma_{R}$

$$
\gamma_{R}=\left(R_{0.01}\right)^{\alpha}
$$

Step 6: Compute the parameter

$$
r_{0.01}=\frac{1}{1+0.78 \sqrt{\frac{L_{G} \gamma_{R}}{f}}-0.38\left(1-\mathrm{e}^{-2 l_{G}}\right)}
$$

Step 7:

$$
\zeta=\tan ^{-1}\left(\frac{h_{R}-h_{s}}{L_{G} r_{0 .}}\right) \quad \text { degrees }
$$

For $\zeta>\theta$

$$
L_{R}=\frac{L_{G} r_{0.01}}{\cos \theta}
$$

Else,

$$
L_{R}=\frac{\left(h_{R}-h_{S}\right)}{\sin \theta} .
$$

Where:

R0.01: average rainfall rate for $0.01 \%$ of one year

$h_{s}$ : height over the average sea of station

$\theta$ : elevation angle

Re: radius of the Earth.

$\varphi$ : latitude of the earth station

$f:$ frequency

This diagram method is one estimation of rainfall attenuation and we can obtain the specific results by the correlation parameters. This diagram method which we use is a simplification version of the ITU standard of recommendation, more specific version can be found in the references $[7,8,9]$.

\section{Analysis of the Results}

\subsection{Receive Power}

From the simulation of the OMNeT++, we change the parameters of the satellite-earth and debug the program to get the results of the simulation. The following table is the specific results of simulation.

Table 1. The parameters and results of the simulation.

\begin{tabular}{lllllll}
\hline $\begin{array}{l}\text { The } 0.01 \% \\
\text { probability of } \\
\text { average annual } \\
\text { rainfall } \\
(\mathrm{mm} / \mathrm{h})\end{array}$ & $\begin{array}{l}\text { Transmit } \\
\text { power }\end{array}$ & $\begin{array}{l}\text { Frequency } \\
(\mathrm{GHz})\end{array}$ & $\begin{array}{l}\text { Angle of } \\
\text { elevation } \\
\text { (degree) }\end{array}$ & $\begin{array}{l}\text { Relative position } \\
\text { between the } \\
\text { satellite and } \\
\text { ground stations } \\
(\mathrm{m})\end{array}$ & $\begin{array}{l}\text { Height of } \\
\text { ground } \\
\text { station } \\
\text { above the }\end{array}$ & $\begin{array}{l}\text { Simulation results } \\
\text { (Received power) }\end{array}$ \\
\hline
\end{tabular}




\begin{tabular}{|c|c|c|c|c|c|c|c|}
\hline & & & & & & $\begin{array}{l}\text { mean sea } \\
\text { level } \\
(\mathrm{km})\end{array}$ & \\
\hline \multirow{6}{*}{$\begin{array}{l}\text { Real physical } \\
\text { propagation } \\
\text { model }\end{array}$} & 10 & 100 & 30 & 60 & 1601932 & 10 & $2.3483491718013963 e-$ \\
\hline & & & & & & & $017 \mathrm{~W}-136.2924 \mathrm{dBm}$ \\
\hline & 15 & 100 & 30 & 60 & 1601932 & 10 & $1.0210363911956929 \mathrm{e}-$ \\
\hline & & & & & & & $017 \mathrm{~W}-139.9096 \mathrm{dBm}$ \\
\hline & 30 & 100 & 30 & 60 & 1601932 & 10 & $3.1272583372063428 \mathrm{e}-$ \\
\hline & & & & & & & $019 \mathrm{~W}-155.0484 \mathrm{dBm}$ \\
\hline $\begin{array}{l}\text { Free space } \\
\text { propagation } \\
\text { model }\end{array}$ & N/A & 100 & 30 & $\mathrm{~N} / \mathrm{A}$ & 1601932 & $\mathrm{~N} / \mathrm{A}$ & $\begin{array}{l}2.4641907908140618 \mathrm{e}- \\
017 \mathrm{~W}-136.0833 \mathrm{dBm}\end{array}$ \\
\hline
\end{tabular}

When we set the parameter of $0.01 \%$ probability of annual rainfall $10 \mathrm{~mm} / \mathrm{h}$, the received power is $-136.2924 \mathrm{dBm}$, as we set the parameter $15 \mathrm{~mm} / \mathrm{h}$, the received power is -139.9096 $\mathrm{dBm}$, as we set the parameter $30 \mathrm{~mm} / \mathrm{h}$, the received power is $-155.0484 \mathrm{dBm}$. When we use the free space propagation model channel, the received power is $-136.0833 \mathrm{dBm}$. From the simulation results, we can see that with the increasing of rainfall, the receive power become less and less with the same transmit power. But the receive power is still acceptable for the receiver and terminals or ground stations.

We put the model of satellite-earth channel into the SGIN, so we can do more research by using this channel model.

We simulate the network packet flow as well, by changing the satellite-earth channel and the free space channel, we obtain different transmission results. As we regard the satellite-earth link as the free space channel, we can get the results of almost no error, as we add the real satellite-earth link channel into the OMNeT++, we are able to get a more specific result.

The statistical diagrams are illustrated in Figure 5, Figure 6, Figure 7, Figure 8 below.

\subsection{Packet Delay and Packet Arrival Rate}

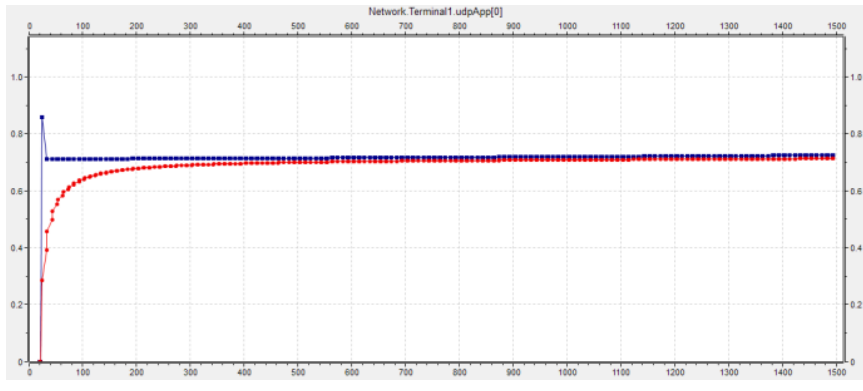

Figure 5. Free space channel packet delay.

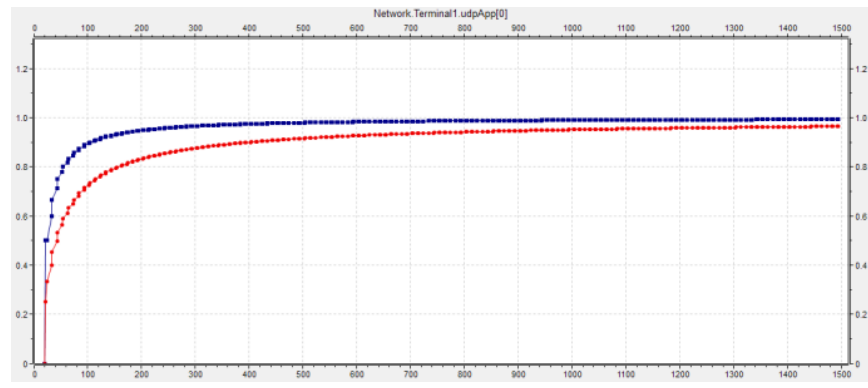

Figure 6. Free space channel packet arrival rate.

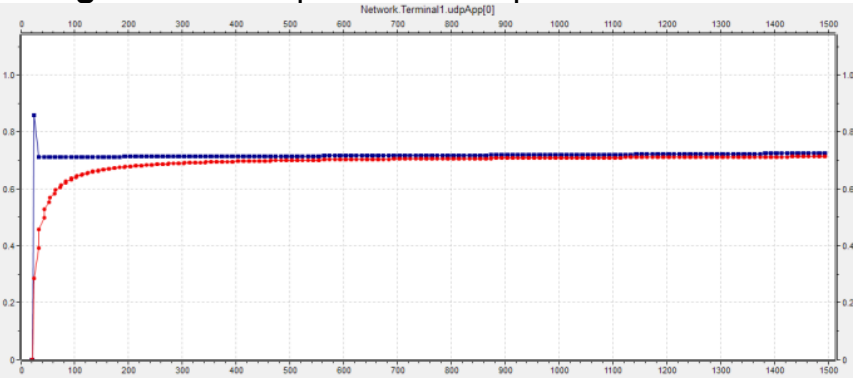

Figure 7. Satellite-earth link channel packet delay.

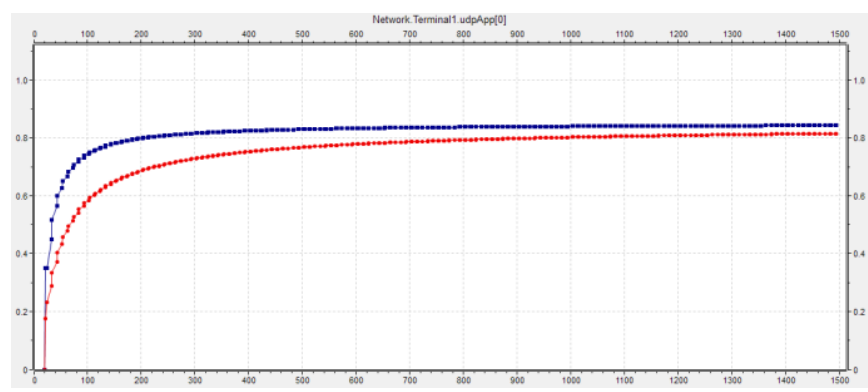

Figure 8. Satellite-earth link channel packet arrival rate. 
From the four figures, we also get the variable results of both the packet delay and the packet arrival rate, the blue curve stands for the instant delay and packet arrival rate while the red curve stands for the mean delay and packet arrival rate. We could see that the packet delay and packet arrival rate become more and more steady as the increasing time and amount of packet. The reason is that the terminals search and get contact with the satellites in the beginning which cost some initial time. Above all, both of the results are acceptable.

From the statistical data, we not only generate the figures, but also get the characteristics of the data. The mean of packet delay of fig5 which we simulate in the free space channel is typically $0.7131 \mathrm{~s}$ (second), and the variance of fig5 is 0.0035 . What is more, the mean of packet arrival rate is typically $96.50 \%$, and the variance of packet arrival rate is 0.0068 . In the simulation of satellite-earth link channel, the mean of packet delay is typically 0.7133 s (second) and the variance of packet delay is 0.0035 , the mean of packet arrival rate is typically $81.50 \%$, and the variance of packet arrival rate is 0.0067 .

\section{Conclusion}

In this paper, we build the structure of network and set up the model of satellite-earth channel link based on OMNeT++, after that, we simulate the network and satellite-earth channel to generate some data and figures that we are interested in, From the results of simulation of space-ground integrated network and satellite-earth link channel, we get the results in the channel with different parameters, and this will be a worthful reference for any communication and network scenario using satellite-earth link channel.

\section{References}

11] LI, F. and YIN, L. (2016) Research status and development trends of security assurance for space-ground integration information network. Journal on Communications volume(37): 156-168.

[2] ZHANG, N. and ZHAO, K. (2015) Thought on constructing the integrated space-terrestrial information network. Journal of CAEIT volume(10): 223-230.

[3] OMNeT++User Guide Version5.0.

[4] OMNeT++Simulation Manual Version5.0.

[5] International Telecommunications Union. http://www.itu.int/dms_pubrec/itu-r/rec/p/R-REC-P.67611-201609-I.pdf.

[6] International Telecommunications Union. http://www.itu.int/dms_pubrec/itu-r/rec/p/R-REC-P.839-4201309-I.pdf.

[7] International Telecommunications Union. http://www.itu.int/dms_pubrec/itu-r/rec/p/R-REC-P.838-3200503-I.pdf.

[8] International Telecommunications Union. http://www.itu.int/dms_pubrec/itu-r/rec/p/R-REC-P.61812-201507-I.pdf.

[9] WANG, ZHU, SUN, et al, A. (2015) Impact of Rain Attenuation of Relay Satellite on Manned Space Flight Mission and Related Solutions. Telecommunication Engineering volume(55): 379-384. 